\title{
Avaliação do impacto de acontecimentos traumáticos na adolescência: validação da Impact of Event Scale-Revised
}

\author{
Assessment of the impact of traumatic events \\ during adolescence: Validation of the \\ Impact of Event Scale-Revised
}

\author{
Marina Isabel Vieira Antunes CUNHA \\ Ana Maria de Jesus XAVIER ${ }^{2}$ \\ Sofia de Miranda ZAGALO'
}

Marcela Salomé Albuquerque Andrade de MATOS$^{2}$

\section{Resumo}

O objetivo do presente estudo foi validar a Impact of Event Scale-Revised em uma amostra de adolescentes portugueses. Esta foi constituída por 383 adolescentes com idades compreendidas entre os 12 e os 18 anos. Foram usados outros questionários, que avaliavam a centralidade da memória de vergonha e sintomatologia psicopatológica. Foram testados vários modelos alternativos da estrutura fatorial da Escala do Impacto do Acontecimento através da Análise Fatorial Confirmatória. Os resultados mostraram que o modelo de três fatores com um fator de segunda ordem tem um melhor ajustamento aos dados. A escala e suas subescalas mostraram uma boa consistência interna, adequada estabilidade temporal e validade convergente com medidas de centralidade da memória de vergonha e sintomas de depressão, ansiedade e estresse. A versão portuguesa da Escala do Impacto do Acontecimento para adolescentes é uma medida válida para avaliar respostas gerais de estresse e/ou impacto traumático, em particular de vivências de vergonha.

Palavras-chave: Adolescente; Análise fatorial confirmatória; Validade dos testes; Vergonha.

\begin{abstract}
The purpose of this paper was to validate the Impact of Event Scale-Revised for Portuguese adolescents. The sample was composed of 383 adolescents aged between 12 and 18 years. Other questionnaires were also used to assess centrality of shame memory and psychopathology symptoms. Alternative factor models of the Impact of Event

"V

${ }^{1}$ Instituto Superior Miguel Torga, Departamento de Psicologia, Programa de Pós-Graduação em Psicologia Clínica. Largo da Cruz de Celas, 1, 3000-132, Coimbra, Portugal. Correspondência para/Correspondence to: M.I.V.A. CUNHA. E-mail: <marina_cunha@ismt.pt>

2 Universidade de Coimbra, Faculdade Psicologia e Ciências da Educação, Centro de Investigação do Núcleo de Estudos e Intervenção Cognitivo-Comportamental. Coimbra, Portugal.

Artigo elaborado a partir da dissertação de S.M. ZAGALO, intitulada "Impacto traumático das experiências de vergonha na adolescência". Instituto Superior Miguel Torga, 2011.
\end{abstract}


Scale-Revised were tested using Confirmatory Factor Analysis. Results showed that the three-factor model with a single second-order factor provided the best fit to the data. The Impact of Event Scale-Revised and its subscales demonstrated good internal consistency and adequate temporal stability and convergent validity with measures of centrality of shame memory and symptoms of depression, anxiety, and stress. The Portuguese version of the Impact of Event Scale-Revised for adolescents is a valid measure to assess general stress responses and/or emotional trauma impact, particularly shame feelings.

Keywords: Adolescent; Confirmatory factor analysis; Validity of tests; Shame.

Na literatura, está bem documentado que os acontecimentos de vida podem ter um papel crucial no ajustamento e bem-estar psicológico das crianças e dos adolescentes (Wolfe \& Mash, 2006). Em particular, os acontecimentos de vida adversos, nomeadamente os estressores ambientais (externos), como, por exemplo, perda, separação parental, doença crônica ou ambientes e práticas parentais de rejeição, frieza ou punição, aumentam a vulnerabilidade para a psicopatologia ao longo do desenvolvimento (Äslund, Nilsson, Starrin, \& Sjöberg, 2007; Cuffe, McKeown, Addy, \& Garrison, 2005; Tangney \& Dearing, 2002; Wolfe \& Mash, 2006).

A investigação em adultos mostra o papel das experiências traumáticas precoces (e.g., abuso sexual, emocional, físico, criticismo, bullying) no desenvolvimento e manutenção das perturbações psicológicas, como a depressão e o estresse pós-traumático (Andrews, 1998; Andrews, Brewin, Rose, \& Kirk, 2000; Hovens et al., 2012; Matos \& Pinto-Gouveia, 2010; Pinto-Gouveia \& Matos, 2011). Na adolescência, período caracterizado por uma maior autonomia em relação aos pais e uma maior aproximação do grupo de pares, os indivíduos tornam-se mais sensíveis às emoções e imagens que criam na mente destes últimos (Gilbert \& Irons, 2009). A possibilidade de gerar afeto negativo no grupo de amigos pode resultar em ser excluído, envergonhado, humilhado ou ridicularizado, o que, por sua vez, pode contribuir para a maturação de um sentido do eu como inferior, sem valor e indesejado (Gilbert \& Irons, 2009).

De fato, alguns estudos demonstram que as experiências traumáticas de bullying e indutoras de sentimentos de vergonha podem aumentar o risco de problemas tanto internalizantes (e.g., depressão e ansiedade) quanto externalizantes (e.g., agressão, comportamentos disruptivos) nessa faixa etária (Äslund et al., 2007; Cuffe et al., 2005; Cunha, Matos, Faria, \& Zagalo, 2012; De Rubeis \& Hollenstein, 2009; Hawker \& Boulton, 2000; Meiser-Stedman, Dalgleish, Yule, \& Smith, 2012; Price, Higa-McMillan, Kim, \& Frueh, 2013; Stuewing \& McCloskey, 2005). Nesse sentido, torna-se importante avaliar e compreender como é que os acontecimentos de vida podem ter um impacto tóxico na saúde mental, visando a promoção da resiliência e a atenuação do impacto das circunstâncias adversas nos jovens.

Um instrumento amplamente usado em estudos empíricos acerca do impacto traumático de uma experiência é a Escala do Impacto do Acontecimento (IES, Impact of Event Scale; Horowitz, Wilner, \& Alvarez, 1979). Esta é uma medida de autorresposta que pode ser usada para avaliar o impacto traumático de qualquer evento de vida específico, avaliando sintomas intrusivos (pesadelos, pensamentos, imagens ou sentimentos intrusivos) e sintomas de evitamento, que consistem na tentativa de diminuir ou escapar de experiências associadas ao evento traumático e ao entorpecimento da resposta (Horowitz et al., 1979).

Posteriormente, a escala foi revista para incluir itens que avaliassem o domínio da hiperativação, de acordo com os critérios de diagnóstico para a Perturbação de Pós-Estresse Traumático que constam na terceira e quarta edição do Diagnostic and Statistical Manual (American Psychiatric Association, 1980). Assim, a Escala do Impacto do Acontecimento - Revista (IES-R, Impact of Event Scale - Revised; Weiss \& Marmar, 1997) passou a ser composta por 22 itens, cotados em uma escala de 5 pontos ( 0 = Nada; 4 = Muitíssimo), que captam as três características primordiais da resposta traumática: 1) intrusão (8 itens; e.g., "Sonhei com isso"); 2) evitamento (8 itens; e.g., "Tentei não falar 
sobre isso"); e 3) hiperativação (6 itens), que avalia sono, irritabilidade, concentração hipervigilância, resposta de ativação e ativação fisiológica (e.g., "Sentia-me irritável e zangado").

No estudo das propriedades psicométricas da IES-R, foram utilizadas duas amostras de profissionais de emergência expostos a situações traumáticas (colapso de autoestrada e terremoto), tendo sido encontrados valores elevados de consistência interna, com coeficientes alfa de Cronbach entre 0,87 e 0,92 para a subescala Intrusão, 0,84 e 0,85 para a subescala Evitamento e 0,79 e 0,90 para a subescala Hiperativação (Weiss \& Marmar, 1997). No que diz respeito à estabilidade temporal, os autores obtiveram igualmente bons valores de teste-reteste (em um período de seis meses de intervalo) para as duas amostras. A Análise de Componentes Principais mostrou a presença de um fator único explicando 49\% da variância. As correlações entre as subescalas foram elevadas: $r=0,74$ entre Intrusão e Evitamento, $r=0,87$ entre Intrusão e Hiperativação e $r=0,87$ entre Evitamento e Hiperativação (Weiss \& Marmar, 1997).

Das diversas pesquisas acerca das características psicométricas da IES- $R$, não existe consenso quanto à dimensionalidade do instrumento. Por exemplo, o estudo conduzido por Wu e Chan (2003) encontrou um único fator com recurso a uma Análise Fatorial Exploratória (AFE). Outros estudos que utilizaram a AFC apontam para a existência de dois (intrusão/hiperativação e evitamento; e.g., Creamer, Bell, \& Failla, 2003) e três fatores (e.g., Beck et al., 2008). Existem ainda outras pesquisas as quais, segundo a utilização da AFC, encontram quatro ou cinco fatores, com um fator de segunda-ordem que engloba o sofrimento geral, atestando a multidimensionalidade da medida (Andrews, Shevlin, Troop, \& Joseph, 2004; Morina et al., 2010).

A IES-R foi usada em vários estudos com crianças e adolescentes para avaliar o impacto da exposição a experiências traumáticas (e.g., guerra) ou vivência de desastres naturais (e.g., terremotos, inundações) (Dyregrov, Kuterovac, \& Barath, 1996; Yule, Bruggencate, \& Joseph, 1994). Mais recentemente, foi desenvolvida uma versão para crianças a partir dos oito anos de idade, designada
"Children's Impact of Event Scale", constituída por 13 itens e três subescalas (evitamento, intrusão, hiperativação) (Deeba, Rapee, \& Prvan, 2014; Smith, Perrin, Dyregrov, \& Yule, 2003).

Para além da inconsistência nos resultados quanto à estrutura fatorial da IES-R, são parcos os estudos na adolescência que avaliam o impacto traumático das experiências de vida em geral e indutoras de vergonha, em particular. A opção pelo estudo de experiências de vergonha enquanto acontecimentos traumáticos deu-se pelo fato de sua ocorrência nesta fase desenvolvimental poder ter um impacto negativo no desenvolvimento posterior do sujeito. Na verdade, nos adolescentes, é frequente um aumento das preocupações relativas à avaliação de si e dos outros, bem como da competição pela aceitação, aprovação e estatuto. Essas características podem aumentar a vulnerabilidade para manifestação de dificuldades de auto-apresentação, medo de rejeição e sentimentos de inferioridade, vivências frequentemente associadas à vergonha (Gilbert \& Irons, 2009). Nesse sentido, assume-se como ponto especialmente importante a avaliação do impacto desse tipo de experiência e das suas potenciais características traumáticas para essa faixa etária.

Dessa maneira, o objetivo deste estudo foi adaptar e analisar as qualidades psicométricas da IES-R em uma amostra de adolescentes portugueses. Foram testados vários modelos alternativos da IES-R através da Análise Fatorial Confirmatória e suas consistências internas e estabilidade temporal foram analisadas. Buscou-se, ainda, analisar a sensibilidade da escala às características sociodemográficas (sexo, idade e escolaridade) dos sujeitos em estudo, bem como explorar a associação do impacto traumático de experiências de vergonha com medidas de centralidade do acontecimento, sintomatologia de ansiedade, depressão e estresse. Teoricamente, espera-se que um acontecimento com um impacto traumático esteja associado à percepção do mesmo como central e ponto de referência para a construção da identidade pessoal e história de vida. Também é esperado que exista uma associação entre os sintomas traumáticos originados pelo acontecimento e os sintomas emocionais negativos (i.e., depressão, ansiedade e estresse). 
Método

\section{Participantes}

Na constituição da amostra, foram tidos em conta os seguintes critérios de inclusão: idades iguais ou superiores a 12 anos e inferiores a 19 anos; escolaridade entre o $7^{\circ}$ e o $12^{\circ}$ ano; e o consentimento por escrito dos encarregados de educação ou representantes legais. A amostra foi constituída por 383 adolescentes, sendo 178 homens (46,5\%) e 205 mulheres $(53,5 \%)$, com idades compreendidas entre os 12 e os 18 anos, $(M=14,93, D P=1,77)$ e que frequentavam o ensino regular $(M=9,20$, $D P=1,60)$ de escolas públicas do distrito de Coimbra, Portugal. Não foram encontradas diferenças estatisticamente significativas de médias de idade em função do sexo, $t(381)=-0,372, p=0,710$.

\section{Instrumentos}

A Escala do Impacto do Acontecimento-Revista (IES-R, Impacto of Event Scale-Revised) (Weiss \& Marmar, 1997) versão Portuguesa para adolescentes (Cunha, Matos, \& Pinto Gouveia, 2011) é constituída por 22 itens, que avaliam os sintomas traumáticos (intrusão, evitamento e hiperativação) como resposta a um acontecimento. Os itens são cotados em uma escala de 5 pontos ( 0 = Nada; 4 = Muitíssimo), sendo que as pontuações elevadas correspondem a níveis mais altos de sintomatologia traumática.

No presente estudo, as instruções da versão original da IES-R foram ligeiramente modificadas de modo a avaliar uma experiência indutora de vergonha e o seu impacto ao longo da vida. Nessa versão, é dada uma breve descrição do que é uma experiência ou emoção de vergonha, sendo posteriormente pedido aos respondentes que recordem uma dessas experiências mais marcantes da sua infância ou adolescência e respondam ao conjunto de itens com base na mesma.

Já a Escala da Centralidade do Acontecimento (CES, Centrality of Event Scale) (Berntsen \&

252 Rubin, 2006) versão portuguesa para adolescentes
(Cunha, Matos, Xavier, \& Faria, 2013) pretende medir até que ponto a memória de um acontecimento estressor representa um ponto de referência central para a identidade pessoal e atribuição de significado a outras experiências de vida. É constituída por 20 itens, cotados em uma escala de 5 pontos ( 1 = Discordo totalmente; 5 = Concordo totalmente).

Alguns exemplos de itens são: "Este acontecimento tornou-se um ponto de referência na forma como eu percebo novas experiências"; "Sinto que este acontecimento se tornou uma parte central da minha história de vida". Quanto maior a pontuação, mais o acontecimento estressor é percebido como central para a identidade pessoal. No presente estudo (como no preenchimento da IES-R), as instruções foram modificadas no sentido de solicitar aos participantes que respondessem o instrumento com base em uma experiência indutora de vergonha marcante e significativa, ocorrida ao longo da vida. Na amostra analisada, obteve-se um alfa de Cronbach de 0,95.

As Escalas de Depressão, Ansiedade e Stress (EADS-21, Depression Anxiety and Stress Scales; Lovibond \& Lovibond, 1995; versão portuguesa Pais-Ribeiro, Honrado, \& Leal, 2004) são um instrumento de autorresposta constituído por 21 itens, os quais avaliam três dimensões de sintomas psicopatológicos: depressão, ansiedade e estresse. Cada item é cotado em uma escala de resposta de 4 pontos ( 0 = "não se aplicou nada a mim"; 3 =" aplicou-se a mim a maior parte das vezes"). No presente estudo, verificaram-se bons índices de consistência interna (depressão: $\alpha=0,89$, ansiedade: $\alpha=0,88$ e estresse: $\alpha=0,90$ ).

\section{Procedimentos}

Para a aplicação da IES-R em adolescentes, o instrumento sofreu pequenas alterações na formulação dos itens no sentido de melhor se adequar à idade pretendida. Com o propósito de salvaguardar os princípios teóricos inerentes à construção do instrumento, foi intencionalmente assumida uma perspetiva conservadora, pelo que a sua estrutura original foi mantida. Posteriormente, foi realizado um estudo piloto com um grupo de 15 adolescentes 
para determinar possíveis dificuldades de compreensão dos itens ou da forma de respondê-los. Não foram apontadas quaisquer dificuldades, originando, assim, a versão para adolescentes. Do ponto de vista conceitual, trata-se do mesmo instrumento, o qual dispõe, agora, de duas versões: uma para adultos (IES-R) e outra para adolescentes (IES-R-A).

As escolas selecionadas são escolas públicas do distrito de Coimbra e as turmas de alunos às quais foram aplicados os questionários iam do $7^{\circ}$ ao $12^{\circ}$ ano e foram escolhidas aleatoriamente. Os questionários foram preenchidos anonimamente, em contexto de sala de aula, sendo que a aplicação durou de 15 a 20 minutos e foi supervisionada por um dos investigadores. No caso do estudo do teste-reteste, foi utilizado um código para preservar o anonimato dos participantes.

Previamente à realização da pesquisa, foi obtida a aprovação por parte do Comitê de Ética da Direção Geral de Ensino de Portugal (Registo $\left.n^{\circ} 0082000004\right)$, bem como solicitada autorização aos diretores de cada escola e o consentimento informado dos jovens e respectivos encarregados de educação. Os participantes foram informados dos objetivos do estudo, confidencialidade e caráter voluntário do mesmo.

No tratamento estatístico dos dados, foi utilizado o Predictive Analytics Software (PAWS Statistics, versão 18.0) e o Amos (versão 18). Foram testados vários modelos de Análise Fatorial Confirmatória por intermédio do software Amos, com o objetivo de comparar a adequação dos diferentes modelos.

Para o estudo da qualidade dos itens e análise da consistência interna, foram usadas estatísticas descritivas para cada item e a correlação item-total foi feita. De acordo com as recomendações de vários autores (Tabachnick \& Fidell, 2007), considerou-se aceitáveis correlações item-total acima de 0,3. Também foi analisada a consistência interna através do alfa de Cronbach, cujo valor pode ser considerado bom quando acima de 0,7 (Tabachnick \& Fidell, 2007).

Foram realizadas análises de correlações de Pearson (Tabachnick \& Fidell, 2007) para avaliar a estabilidade temporal da escala, a influência das variáveis idade e escolaridade, bem como a validade convergente da IES-R-A com outras medidas de centralidade do acontecimento e sintomas de depressão, ansiedade e estresse. Utilizou-se, ainda, testes t - Student para amostras independentes (Tabachnick \& Fidell, 2007) a fim de comparar as pontuações médias de impacto traumático das experiências de vergonha entre homens e mulheres. A magnitude da diferença de médias (magnitude do efeito) foi verificada através do $d$ de Cohen (Cohen, 1988). De acordo com o autor, convencionou-se que os valores de $d$ são considerados pequenos (se $0,20 \leq d<0,50$ ); médios (se 0,50 $\leq d<0,80$ ); e grandes (se $d \geq 0,80$ ).

\section{Resultados}

\section{Análise preliminar dos dados}

Foram analisados os pressupostos de normalidade multivariada através do Teste de Kolmogorov-Smirnov e do exame dos coeficientes de assimetria e curtose. Nenhuma variável apresentou valores de assimetria e de curtose indicadores de violações severas à distribuição normal $(S k<3$ and $K u<8$ - 10) (Kline, 2005). A existência de outliers foi avaliada pela distância quadrada de Mahalanobis $\left(D^{2}\right)$, que indicou a presença de alguns valores extremos, mas optou-se pela manutenção dos mesmos para não diminuir a variabilidade associada ao fator em estudo e não limitar uma interpretação relevante na análise. Os valores ausentes ("missing completely at random") foram tratados através da técnica de substituição pela média.

\section{Modelos de Análise Fatorial Confirmatória}

Neste estudo, através de AFC, foram comparados os seguintes modelos (Tabela 1): (a) Modelo 1: um único fator; (b) Modelo 2: dois fatores latentes intercorrelacionados: (1) evitamento, com oito itens, (2) intrusão e hiperativação, com catorze itens; (c) Modelo 3: três fatores latentes intercorrelacionados

(1) evitamento, com oito itens, (2) intrusão, com 
oito itens, e (3) hiperativação, com seis itens; (d) Modelo 4: modelo hierárquico de segunda ordem, com um fator latente global de IES "Impacto Traumático" e 22 variáveis manifestas. O método de estimação do modelo utilizado foi o Método de Máxima Verosimilhança (ML, Maximum Likelihood).

Para analisar a qualidade global das AFC, observou-se o teste de qui-quadrado $\left(\chi^{2}\right)$, cujo valor precisaria ser o menor possível, e o nível de significância associado, o qual deveria ser superior a 0,05 (Kline, 2005). Uma vez que esse teste estatístico é muito sensível ao tamanho da amostra e a afastamentos à normalidade e linearidade (Schermelleh-Engel, Moosbrugger, \& Müller, 2003), foram ainda utilizados, em simultâneo, outros índices de qualidade de ajustamento: Goodness of Fit Index (GFI); Comparative Fit Index (CFI); Tucker-Lewis Index (TLI), Root Mean Square Error of Approximation (RMSEA). Considerou-se que valores de GFI, CFI e TLI iguais ou superiores a 0,90 correspondem a um bom ajustamento dos dados; valores de RMSEA inferiores a 0,05 são indicadores de um excelente ajustamento, iguais ou inferiores a 0,08 revelam um bom ajustamento e iguais ou inferiores a 0,10 apontam para um ajustamento marginal (Kline, 2005; Marôco, 2010).

A reespecificação do modelo foi feita a partir dos índices de modificação (superiores a 11; $p<0.001$ ) produzidos pelo software Amos, respeitando considerações teóricas (i.e., conteúdo dos itens). Para efeitos de comparação dos modelos, recorreu-se ao (M)ECVI (Expected Cross-Validation Index), indicando que o modelo com menor valor de (M)ECVI é o melhor e mais estável na população (Marôco, 2010).
A qualidade do ajustamento local foi avaliada pelos pesos fatoriais $(\lambda \geq 0,5)$ e pela fiabilidade individual dos itens $\left(R^{2} \geq 0,25\right)$ (Marôco, 2010). A Fiabilidade Compósita (FC) indica a consistência interna dos itens que refletem o fator, considerando-se valores de $F C \geq 0,7$ indicadores de uma adequada fiabilidade de constructo (Marôco, 2010).

Como se pode observar pela Tabela 1, os resultados mostram que, de um modo geral, os vários modelos testados apresentam índices de ajustamento sofríveis. Verifica-se, ainda, que o modelo 3, no qual foram testados três fatores latentes intercorrelacionados ( 1 - evitamento, 2 - intrusão, 3 - hiperativação), apresentou um valor menor de (M)ECVI, comparativamente aos outros modelos. Procedeu-se, em seguida, a análise dos índices de modificação, com o objetivo de melhorar o ajustamento do referido modelo. Foram correlacionados os erros de medida dos seguintes itens: 17 (e17) e 22 (e22); 11 (e11) e 17 (e17); 2 (e2) e 6 (e6); e dos itens 5 (e5) e 11 (e11). Essas correlações também se justificam, de um ponto de vista teórico, pela semelhança do conteúdo dos itens pertencentes ao mesmo fator. Assim, o modelo 3 reespecificado apresenta um ajustamento aos dados adequado, com valores de CFI e RMSEA dentro dos valores de corte $(C F I \geq 0,90$ e RMSEA $\leq 0,08)$, embora os índices restantes permaneçam sofríveis. Adicionalmente, através do teste de diferenças de Qui-quadrado, verificou-se que o modelo re-especificado apresenta uma qualidade de ajustamento significativamente superior à do modelo original na amostra em estudo $\left(\chi_{(4)}^{2}=171,873, p<0,05>\chi^{20.95 ;(4)}=9.488\right)$, bem

Tabela 1

Comparação dos índices de ajustamento dos modelos testados através de Análise Fatorial Confirmatória

\begin{tabular}{|c|c|c|c|c|c|c|c|}
\hline Modelos testados & $\chi^{2}$ & $g l$ & GFI & TLI & $\mathrm{CFI}$ & RMSEA & MECVI \\
\hline Modelo 1 (1 fator) & $973,546^{*}$ & 209 & 0,79 & 0,81 & 0,83 & 0,098 & 2,794 \\
\hline Modelo 2 (2 fatores: Evitamento vs. Intrusão-Hiperativação) & $845,826^{*}$ & 208 & 0,82 & 0,84 & 0,86 & 0,090 & 2,465 \\
\hline Modelo 3 (três fatores) & $836,204^{*}$ & 206 & 0,82 & 0,84 & 0,86 & 0,089 & 2,451 \\
\hline Modelo 3 reespecificado & $664,311^{*}$ & 202 & 0,86 & 0,88 & 0,90 & 0,077 & 2,023 \\
\hline Modelo 4 segunda ordem & $664,311^{*}$ & 202 & 0,86 & 0,88 & 0,90 & 0,077 & 2,023 \\
\hline
\end{tabular}

Nota: ${ }^{*} p \leq 0.001$.

GFI: Goodness of Fit Index; TLl: Tucker-Lewis Index; CFI: Comparative Fit Index; RMSEA: Root Mean Square Error of Approximation; MECVI: Expected 
como um (M)ECVI consideravelmente menor $(2,023$ versus 2,451).

Quanto à qualidade de ajustamento local do modelo, verificou-se que todos os itens apresentam pesos fatoriais elevados, que variam entre 0,50 (item 11) e 0,79 (item 16), e fiabilidades individuais igualmente elevadas, as quais vão de 0,25 (item 11) a 0,63 (item 16) (Tabela 2). A fiabilidade compósita dos fatores do modelo reespecificado revelou-se elevada, sendo de 0,84 para o fator Evitamento (8 itens), 0,91 para Intrusão (8 itens) e 0,80 para Hiperativação (6 itens). A correlação entre eles é elevada: "Evitamento" e "Intrusão" apresentam uma correlação de $r=0,88(p<0,001)$, "Evitamento" e "Hiperativação" apresentam uma correlação de $r=0,85(p<0,001)$ e os fatores "Intrusão" e "Hiperativação" apresentam uma correlação de $r=0,97(p<0,001)$.

\section{Análise Fatorial de Segunda Ordem}

De acordo com os princípios da análise fatorial, os dados anteriormente obtidos sugerem a existência de fatores hierárquicos superiores. Desse modo, foi testado um modelo de segunda ordem designado "Impacto Traumático", uma vez que (i) a conceitualização teórica acerca do trauma prevê a existência de um fator latente de impacto traumático global, e (ii) as correlações significativas observadas entre as três dimensões da IES podem dever-se à existência de um fator latente de segunda ordem. Partindo do modelo re-especificado anteriormente do IES-R-A (Modelo 3 reespecificado), testou-se um modelo hierárquico de segunda ordem com um fator latente global de Impacto Traumático.

Os índices de ajustamento estão apresentados na Tabela 1. Os resultados indicam ainda que os caminhos do fator de segunda ordem "Impacto

Tabela 2

Pesos de regressão estandardizados $(\lambda)$ e Correlações múltiplas ao quadrado $\left(R^{2}\right)$ para os itens considerados no modelo tridimensional da Impact of Event Scale - Revised ( $N=383$ )

\begin{tabular}{|c|c|c|}
\hline Item & $\lambda$ & $R^{2}$ \\
\hline \multicolumn{3}{|l|}{ Evitamento } \\
\hline 5. Tentei não ficar perturbado quando pensava nisso ou era lembrado disso. & 0,52 & 0,27 \\
\hline 7. Senti como se aquilo não tivesse acontecido ou não fosse real. & 0,62 & 0,39 \\
\hline 8. Evitei estar perto de coisas que me lembrassem disso. & 0,63 & 0,40 \\
\hline 11. Tentei não pensar no acontecimento. & 0,50 & 0,25 \\
\hline 12. Tinha consciência que ainda tinha muitos sentimentos sobre isso, mas não lidava com eles. & 0,75 & 0,56 \\
\hline 13. Sentia-me como se estivesse anestesiado em relação a isso. & 0,60 & 0,36 \\
\hline 17. Tentei tirar isso da memória. & 0,54 & 0,29 \\
\hline 22. Tentei não falar sobre isso. & 0,61 & 0,37 \\
\hline \multicolumn{3}{|l|}{ Intrusão } \\
\hline 1. Qualquer coisa que me lembrasse do acontecimento trazia de volta sentimentos sobre isso. & 0,68 & 0,46 \\
\hline 2. Tive dificuldades em permanecer a dormir. & 0,70 & 0,49 \\
\hline 3. Outras coisas persistiam em fazer-me pensar naquilo. & 0,76 & 0,58 \\
\hline 6. Pensei sobre isso quando não era minha intenção. & 0,69 & 0,48 \\
\hline 9. Imagens do acontecimento vinham-me à cabeça. & 0,75 & 0,57 \\
\hline 14. Dei por mim a agir ou sentir como se estivesse de novo naquela situação. & 0,68 & 0,46 \\
\hline 16. Tive ondas de sentimentos intensos em relação ao acontecimento. & 0,79 & 0,63 \\
\hline 20. Sonhei com isso. & 0,70 & 0,49 \\
\hline \multicolumn{3}{|l|}{ Hiperativação } \\
\hline 4. Sentia-me irritável e zangado. & 0,64 & 0,41 \\
\hline 10. Estava agitado e ficava nervoso com facilidade. & 0,78 & 0,61 \\
\hline 15. Tive dificuldades em adormecer. & 0,75 & 0,56 \\
\hline 18. Tive dificuldades em me concentrar. & 0,76 & 0,57 \\
\hline $\begin{array}{l}\text { 19. Coisas que me lembravam o acontecimento provocavam-me reações físicas, como transpiração, dificuldades em respirar, } \\
\text { enjoos, palpitações. }\end{array}$ & 0,70 & 0,49 \\
\hline 21. Senti-me alerta e vigilante. & 0,67 & 0,45 \\
\hline
\end{tabular}


Traumático" para os respetivos fatores são significativos e apresentam pesos fatoriais elevados, nomeadamente do IES global para Evitamento $\lambda=0,88$, do IES global para Intrusão $\lambda=1,00$ e do IES global para Hiperativação $\lambda=0,97$. As correlações múltiplas ao quadrado $\left(R^{2}\right)$ foram $R^{2}=0,77$ para Evitamento, $R^{2}=0,99$ para Intrusão e $R^{2}=0,94$ para Hiperativação. A adição do fator de segunda ordem é apoiada pela estrutura correlacional observada na amostra sob estudo.

\section{Fidelidade}

\section{Análise dos itens e consistência interna}

Pela análise da qualidade dos itens da Escala do Impacto do Acontecimento - Revista para Adolescentes, verifica-se que todos os itens apresentam correlações item-total iguais ou superiores a 0,45, o que indica que contribuem consistentemente para a medida global de impacto traumático. As três subescalas apresentam elevados coeficientes alfa de Cronbach (Evitamento: $\alpha=0,84$; Hiperativação: $\alpha=0,86$; Intrusão: $\alpha=0,89$ ) e a escala total apresenta um excelente coeficiente alfa de Cronbach $(\alpha=0,94)$, atestando uma excelente consistência interna (Tabachnick \& Fidell, 2007). Adicionalmente, todos os itens contribuem para a consistência interna do instrumento, já que a remoção de algum item não incrementaria os valores de alfa de Cronbach. A escala apresentou os seguintes valores médios: Total da escala, $M=34,72, D P=18,90, I C$ $95 \%[32,62,36,62]$; Evitamento, $M=13,34$, $D P=7,22$, IC 95\% [13,61, 15,06]; Hiperativação, $M=8,28, D P=6,04, I C$ 95\% $[7,67,8,88]$ e Intru- são, $M=12,10, D P=7,44, I C 95 \%[11,35,12,85]$ (Tabela 3).

\section{Fidelidade teste-reteste}

Para analisar a estabilidade temporal da IESR-A, foi necessário administrá-la em dois momentos diferentes, com um intervalo de três semanas entre si, a um grupo de adolescentes $(N=18)$. Obteve-se, assim, um coeficiente de correlação de $r$ de Pearson de 0,67 ( $p=0,002)$, o que sugere uma adequada estabilidade temporal do instrumento.

\section{Influência das variáveis sexo, idade e anos de escolaridade}

Os resultados obtidos na IES-R-A global e respectivas subescalas não se mostraram associados à idade e aos anos de escolaridade. Verificaram-se diferenças estatisticamente significativas entre os sexos no que diz respeito à pontuação total da IES-R-A, $t(381)=-4,505, p<0,001$, à subescala Evitamento, $t(381)=-4,853, p<0,001$, à subescala Intrusão, $t(381)=-4,522, p<0,001$, e à subescala Hiperativação, $t(381)=-2,706, p=0,007$. Quer para a escala total da IES-R, quer para as suas dimensões, as mulheres apresentam, em média, pontuações mais elevadas do que os homens (IES-R-A total: $M_{\text {feminino }}=38,67$, versus $M_{\text {masculino }}=30,16$; Evitamento: $M_{\text {feminino }}=15,96$, versus $M_{\text {masculino }}=12,47$; Intrusão: $M_{\text {feminino }}=13,66$, versus $M_{\text {masculino }}=10,30$; Hiperativação: $M_{\text {feminino }}=9,05$, versus $M_{\text {masculino }}=7,39$ ). O tamanho do efeito, calculado através do $d$ de Cohen, variou entre 0,28 e 0,50, considerados por Cohen (1988) pequenos e moderados, respectivamente.

Tabela 3

Média, Desvio-Padrão, Mínimo e Máximo, medidas de Assimetria e Curtose, e Intervalos de Confiança para a Escala do Impacto do Acontecimento e subescalas $(N=383)$

\begin{tabular}{|c|c|c|c|c|c|c|c|}
\hline Variáveis & Média & Desvio-Padrão & Mínimo & Máximo & Assimetria & Curtose & IC95\% \\
\hline IES-R & 34,72 & 18,90 & 0 & 78 & 0,250 & $-0,752$ & $32,82-36,62$ \\
\hline Intrusão & 12,10 & 7,49 & 0 & 29 & 0,357 & $-0,883$ & $11,35-12,85$ \\
\hline Evitamento & 14,34 & 7,22 & 0 & 32 & 0,044 & $-0,651$ & $13,61-15,06$ \\
\hline Hiperativação & 8,28 & 6,04 & 0 & 24 & 0,516 & $-0,675$ & $7,67-\quad 8,88$ \\
\hline
\end{tabular}

Nota: IES-R: Impact of Event Scale - Revised; IC95\%: Intervalos de Confiança de 95\%. 


\section{Validade convergente}

Uma vez que os resultados anteriores mostraram diferenças em função do sexo, foi controlado o efeito desta variável na exploração da matriz de correlações entre a IES-R-A total, as suas subescalas, a centralidade das memórias de vergonha (CES) e as três escalas de sintomatologia psicopatológica (EADS-21) (Tabela 4). De um modo geral, os resultados mostram correlações significativas e positivas entre o impacto traumático da experiência de vergonha (IES-R-A) e a centralidade do acontecimento (CES). Existem, ainda, correlações significativas e positivas entre o impacto traumático da experiência de vergonha (IES-R-A) e os sintomas de depressão, ansiedade e estresse (EADS-21).

\section{Discussão}

Este estudo testou vários modelos alternativos da estrutura fatorial da IES-R-A, através da AFC, em uma amostra de adolescentes da comunidade. Os resultados indicam que o modelo de três fatores intercorrelacionados, constituído pelas dimensões Evitamento, Intrusão e Hiperativação, apresenta um ajustamento melhor aos dados, comparativamente aos outros modelos testados, apesar de alguns índices globais de ajustamento permanecerem sofríveis. Além disso, um modelo de segunda ordem, com um fator latente de "Impacto Traumático" explicando a associação entre os três fatores de primeira ordem da IES-R-A, mostrou-se plausível, dadas as correlações significativas e elevadas entre as três dimensões da IES-R-A.
Esses resultados estão de acordo com outros estudos acerca da estrutura multidimensional da IES-R (Andrews et al., 2004; Beck et al., 2008; Deeba et al., 2014), sugerindo a necessidade de encontrar um suporte empírico robusto, com amostras representativas para os componentes de intrusão, hiperativação e evitamento da IES-R.

Com relação à fidelidade do instrumento para adolescentes, os resultados mostram uma boa consistência interna, quer para o total da escala quer para as três subescalas. Revela, ainda, uma adequada estabilidade temporal. Esses dados são semelhantes aos encontrados em outras pesquisas sobre as características psicométricas da IES-R (Matos, Pinto-Gouveia, \& Martins, 2011; Weiss \& Marmar, 1997). Neste estudo, o sexo parece ter um efeito na percepção do acontecimento, sendo que, nos adolescentes, as experiências indutoras de vergonha tendem a ter um maior impacto traumático (com características de evitamento, intrusão e hiperativação) nas mulheres. A literatura corrobora esses resultados, mostrando que o sexo feminino apresenta maior risco do que o masculino para desenvolver sintomas traumáticos e perturbação pós-estresse traumático (Deeba et al., 2014; Olff, Langeland, Draijer, \& Gersons, 2007; Yule et al. 1994).

Por outro lado, estudos sobre vivências de vergonha em adolescentes têm evidenciado resultados mistos com relação às diferenças de sexo (Cunha et al., 2012). Quando são encontradas diferenças significativas entre homens e mulheres, verifica-se que são estas que relatam mais sentimentos de vergonha (De Rubeis \& Hollenstein, 2009). Esse

Tabela 4

Correlações de Pearson (two-tailed) entre a IES e suas subescalas ( $N=383)$ e a CES $(n=358)$ e a EADS-21 $(n=369)$, controlando o sexo

\begin{tabular}{|c|c|c|c|c|c|c|c|c|}
\hline \multirow{2}{*}{ Variáveis } & \multicolumn{4}{|c|}{ Homens $(n=178)$} & \multicolumn{4}{|c|}{ Mulheres $(n=205)$} \\
\hline & IES T. & Evit. & Intr. & Hiper. & IES T. & Evit. & Intr. & Hiper. \\
\hline CES & 0,44 & 0,32 & 0,47 & 0,44 & 0,47 & 0,34 & 0,50 & 0,43 \\
\hline Depressão (EADS-21) & 0,66 & 0,63 & 0,63 & 0,69 & 0,58 & 0,42 & 0,54 & 0,60 \\
\hline Ansiedade (EADS-21) & 0,62 & 0,48 & 0,61 & 0,64 & 0,66 & 0,46 & 0,63 & 0,70 \\
\hline Estresse (EADS-21) & 0,62 & 0,52 & 0,58 & 0,62 & 0,55 & 0,35 & 0,53 & 0,61 \\
\hline
\end{tabular}

Nota: Todas as correlações são significativas, com um nível de significância de $p<0,001$.

IES T.: Escala do Impacto do Acontecimento Total; Evit.: Subescala Evitamento; Intr.: Subescala Intrusão; Hiper.: Subescala Hiperativação; CES: Escala da Centralidade do Acontecimento; EADS-21: Escalas de Depressão Ansiedade e Estresse; IES: Impact of Event Scale. 
dado pode, assim, ajudar a explicar o fato das mulheres reportarem níveis mais elevados de sintomas traumáticos.

De um modo geral, os resultados indicam que os adolescentes cujas experiências de vergonha tiveram um impacto traumático tendem a perceber essas experiências como centrais para a sua vida e identidade, bem como a manifestar níveis mais elevados de sintomas de depressão, ansiedade e estresse. Esses dados estão em consonância com os obtidos nos estudos de Matos et al. (2011).

Não obstante a contribuição da presente pesquisa ao disponibilizar um instrumento útil e fidedigno para a investigação e prática clínica com jovens, apresenta, porém, duas grandes limitações: (i) apesar dos índices globais de AFC sugerirem um aceitável ajustamento dos dados ao modelo de três fatores, a maioria dos índices não atinge o ponto de corte indicador de um excelente ajustamento; (ii) os participantes pertencem à comunidade e as instruções foram modificadas para avaliar especificamente uma experiência indutora de vergonha, pelo que os resultados não podem ser generalizados para outras amostras com diferentes tipos de acontecimentos traumáticos em diferentes contextos.

Assim, recomenda-se cautela na interpretação dos resultados e no uso da pontuação total da IES-R em amostras da comunidade. Poderá ser igualmente útil analisar os fatores da IES-R atendendo aos objetivos específicos de cada investigação. Importante ressaltar, ainda, a necessidade de estudos futuros sobre a adaptação do instrumento aos novos critérios de diagnóstico indicados pelo DSM-5 (American Psychiatric Association, 2014). É também recomendável a replicação do estudo da estabilidade temporal da escala em uma amostra mais ampla. Conclui-se, dessa maneira, que o presente estudo mostrou ser a versão portuguesa da IES-R para adolescentes uma medida válida para avaliar respostas gerais de estresse e/ou impacto traumático, em particular de experiências indutoras de vergonha.

\section{Colaboradores}

M.I.V.A CUNHA participou da concepção, na coleta e análise dos dados e na redação do artigo. A.M.J.
XAVIER e S.M. ZAGALO colaboraram na análise dos dados, na redação e na revisão do artigo. M.S.A.A. MATOS colaborou na redação e na revisão do artigo.

\section{Referências}

American Psychiatric Association. (1980). Diagnostic and statistical manual of mental disorders (3rd ed., DSMIII). Washington, D.C.: Author.

American Psychiatric Association. (2014). Manual de diagnóstico e estatística das perturbações mentais (5a ed., DSM-V). Lisboa: Climepsi.

Andrews, B. (1998). Shame and childhood abuse. In P. Gilbert \& B. Andrews (Eds.), Shame: Interpersonal behavior, psychopathology, and culture (pp.3-38). Oxford: Oxford University Press.

Andrews, B., Brewin, C. R., Rose, S., \& Kirk, M. (2000). Predicting PTSD symptoms in victims of violent crime: The role of shame, anger, and childhood abuse. Journal of Abnormal Psychology, 109(1), 69-73. https:// doi.org/10.1037/0021-843X.109.1.69

Andrews, L., Shevlin, M., Troop, N., \& Joseph, S. (2004). Multidimensionality of intrusion and avoidance: Alternative factor models of the Impact of Event Scale. Personality and Individual Differences, 36(2), 431-446. https://doi.org/10.1016/S0191-8869(03)00107-7

Äslund, C., Nilsson, K. W., Starrin, B., \& Sjöberg, R. L. (2007). Shaming experiences and the association between adolescent depression and psychosocial risk factors. European Child \& Adolescent Psychiatry, 16(5), 298-304. https://doi.org/10.1007/s00787-006-0 564-1

Beck, J. G., Grant, D. M., Read, J. P., Clapp, J. D., Coffey, S. F., Miller, L. M., \& Palyo, S. A. (2008). The Impact of Event Scale-Revised: Psychometric properties in a sample of motor vehicle accident survivors. Journal of Anxiety Disorders, 22(2), 187-198. https://doi.org/ 10.1016/j.janxdis.2007.02.007

Berntsen, D., \& Rubin, D. C. (2006). The centrality of event scale: A measure of integrating a trauma into one's identity and its relation to post-traumatic stress disorder symptoms. Behaviour Research and Therapy, 44(2), 219-231. https://doi.org/10.1016/j.brat. 2005.01.009

Cohen, J. (1988). Statistical power analysis for the behavioral sciences (2nd ed.). Hillsdale: Lawrence Erlbaum Associates.

Creamer, M., Bell, R., \& Failla, S. (2003). Psychometric properties of the impact of event scale-revised. Behaviour Research and Therapy, 41(12), 1489-1496. https://doi.org/10.1016/j.brat.2003.07.010

Cuffe, S. P., McKeown, R. E., Addy, C. L., \& Garrison, C. Z. (2005). Family and psychosocial risk factors in a longitudinal epidemiological study of adolescents. 
Journal of the American Academy of Child and Adolescent Psychiatry, 44(2), 121-129. https://doi.org/ 10.1097/00004583-200502000-00004

Cunha, M., Matos, M., \& Pinto Gouveia, J. (2011). Tradução e adaptação da "A Escala do Impacto do Acontecimento (IES-R)" para adolescentes. Universidade de Coimbra, Portugal. [Manuscrito não-publicado].

Cunha, M., Matos, M., Faria, D., \& Zagalo, S. (2012). Shame memories and psychopathology in adolescence: The mediator effect of shame. International Journal of Psychology \& Psychological Therapy, 12(2), 203-218.

Cunha, M., Matos, M., Xavier, A., \& Faria, D. (2013). The adolescents' version of the event centrality scale (CESA): Study of its psychometric properties. Atentión Primaria, 45 (Especial Congreso I), 163. Retrived October 5, 2015, from http://apps.elsevier.es/ watermark/ctl_servlet?_f $=10 \&$ pident_articulo $=902$ 01512 \&pident_usuario $=0$ \&pcontactid $=\&$ pident revista $=27 \&$ ty $=38$ \&accion $=$ L\&origen $=$ zonadelectura $\&$ web $=w w w$.elsevier.es\&lan $=$ en \&fichero $=27 v 45 n$ Esp.Congresoa90201512pdf001.pdf

De Rubeis, S., \& Hollenstein, T. (2009). Individual differences in shame and depressive symptoms during early adolescence. Personality and Individual Differences, 46, 477-482. https://doi.org/10.1016/j. paid.2008.11.019

Deeba, F., Rapee R. M., \& Prvan, T. (2014). Psychometric properties of the Children's Revised Impact of Events Scale (CRIES) with Bangladeshi children and adolescents. PeerJ, 2(536), 2-13. https://doi.org/10. 7717/peerj.536

Dyregrov, A., Kuterovac, G., \& Barath, A. (1996). Factor analysis of the impact of event scale with children in war. Scandinavian Journal of Psychology, 37(4), 339-350.

Gilbert, P., \& Irons, C. (2009). Shame, self-criticism and self-compassion in adolescence. In N. B. Allen \& L. B. Sheeber (Eds.), Adolescent emotional development and the emergence of depressive disorders (pp.195-214). Cambridge: Cambridge University Press. https://doi. org/10.1017/CBO9780511551963

Hawker, D. S. J., \& Boulton, M. J. (2000), Twenty years' research on peer victimization and psychosocial maladjustment: A meta-analytic review of crosssectional studies. Journal of Child Psychology and Psychiatry, 41, 441-455. https://doi.org/10.1111/14 69-7610.00629

Horowitz, M., Wilner, N., \& Alvarez, W. (1979). Impact of Event Scale: A measure of subjective stress. Psychosomatic Medicine, 41(3), 209-218.

Hovens, J. G. F. M., Giltay, E. J., Wiersma, J. E., Spinhoven, P., Penninx, B. W. J. H., \& Zitman, F. G. (2012). Impact of childhood life events and trauma on the course of depressive and anxiety disorders. Acta Psychiatrica Scandinavica, 126(3), 198-207. https://doi.org/10. 1111/j.1600-0447.2011.01828.x
Kline, R. B. (2005). Principles and practice of structural equation modeling (2nd ed.). New York: Guilford.

Lovibond, P., \& Lovibond, H. (1995). The structure of negative emotional states: Comparison of the Depression Anxiety Stress Scales (DASS) with Beck Depressive and Anxiety Inventories. Behaviour Research and Therapy, 33(3), 335-343. https://doi.org/ 10.1016/0005-7967(94)00075-U

Marôco, J. (2010). Análise de equações estruturais: fundamentos teóricos, software \& aplicações. Lisboa: Report Number.

Matos, M., \& Pinto Gouveia, J. (2010). Shame as a traumatic memory. Clinical Psychology \& Psychotherapy, 17(4), 299-312. https://doi.org/10.10 02/cpp.659

Matos, M., Pinto-Gouveia, J., \& Martins, S. (2011). O impacto traumático de experiências de vergonha: estudo das propriedades psicométricas da versão portuguesa da Impact of Event Scale - Revised. Psychologica, 54, 413-438. Recuperado em outubro 5, 2015, de http:// iduc.uc.pt/index.php/psychologica/article/view/1114

Meiser-Stedman, R., Dalgleish, T., Yule, W., \& Smith, P. (2012). Intrusive memories and depression following recent non-traumatic negative life events in adolescents. Journal of Affective Disorders, 137(1), 70-78. https://doi.org/10.1016/j.jad.2011.12.020

Morina, N., Böhme, H. F., Ajdukovic, D., Bogic, M., Franciskovic, T., Galeazzi, G. M., ... Priebe, S. (2010). The structure of post-traumatic stress symptoms in survivors of war: Confirmatory factor analyses of the Impact of Event Scale - Revised. Journal of Anxiety Disorders, 24(6), 606-611. https://doi.org/10.1016/ j.janxdis.2010.04.001

Olff, M., Langeland, W., Draijer, N., \& Gersons, B. P. (2007). Gender differences in posttraumatic stress disorder. Psychological Bulletin, 133(2), 183. https:// doi.org/10.1037/0033-2909.133.2.183

Pais-Ribeiro, J. L., Honrado, A., \& Leal, I. (2004). Contribuição para o estudo da adaptação portuguesa das escalas de ansiedade, depressão e stress (EADS) de 21 Itens de Lovibond e Lovibond. Psicologia, Saúde \& Doença, 5(2), 229-239. Recuperado em outubro 5, 2015, de http://www.scielo.mec.pt/pdf/psd/v5n2/ v5n2a07.pdf

Pinto-Gouveia, J., \& Matos, M. (2011). Can shame memories become a key to identity? The centrality of shame memories predicts psychopathology. Applied Cognitive Psychology, 25(2), 281-290. https://doi.org/ 10.1002/acp.1689

Price, M., Higa-McMillan, C., Kim, S., \& Frueh, B. C. (2013). Trauma experience in children and adolescents: An assessment of the effects of trauma type and role of interpersonal proximity. Journal of Anxiety Disorders, 27(7), 652-660. https://doi.org/10.1016/ j.janxdis.2013.07.009 
Schermelleh-Engel, K., Moosbrugger, H., \& Müller, H. (2003). Evaluating the fit of structural equation models: Tests of significance and descriptive goodnessof-fit measures. Methods of Psychological Research Online, 8(2), 23-74.

Smith, P., Perrin, S., Dyregrov, A., \& Yule, W. (2003). Principal components analysis of the impact of event scale with children in war. Personality and Individual Differences, 34(2), 315-322. https://doi.org/10.1016/ S0191-8869(02)00047-8

Stuewig, J., \& McCloskey, L. A. (2005). The relation of child maltreatment to shame and guilt among adolescents: Psychological routes to depression and delinquency. Child Maltreatment, 10(4), 324-336. https://doi.org/10.1177/1077559505279308

Tabachnick, B. G., \& Fidell, L. S. (2007). Using multivariate statistics (5th ed.). Boston: Allyn and Bacon.

Tangney, J. P., \& Dearing, R. L. (2002). Shame and guilt. New York: Guilford Press.
Weiss, D. S., \& Marmar, C. R. (1997). The Impact of Event Scale - Revised. In J. P. Wilson \& T. M. Keane (Eds.), Assessing psychological trauma and PTSD (pp.399-411). New York: Guilford Press.

Wolfe, D. A., \& Mash, E. J. (2006). Behavioral and emotional disorders in adolescents. London: Guilford Press.

Wu, K. K., \& Chan, K. S. (2003). The development of the Chinese version of Impact of Event Scale - Revised (CIES-R). Social Psychiatry and Psychiatric Epidemiology, 38(2), 94-98. https://doi.org/10.1007/s00127-003-0 $611-x$

Yule, W., Bruggencate, S. T., \& Joseph, S. (1994). Principal components analysis of the Impact of Events Scale in adolescents who survived a shipping disaster. Personality and Individual Differences, 16(5), 685-691. https://doi.org/10.1016/0191-8869(94)90210-0

Recebido: novembro 18, 2014

Versão final: outubro 16, 2015

Aprovado: fevereiro 16, 2016 